\title{
Excitation functions of alpha-induced nuclear reactions on natural erbium and natural ytterbium targets
}

\author{
B. Király ${ }^{1, a}$, F. Tárkányi ${ }^{1}$, S. Takács ${ }^{1}$, A. Hermanne ${ }^{2}$, S.F. Kovalev ${ }^{3}$, and A.V. Ignatyuk ${ }^{3}$ \\ ${ }^{1}$ Institute of Nuclear Research of the Hungarian Academy of Sciences (ATOMKI), 4026 Debrecen, Hungary \\ 2 Cyclotron Laboratory, Vrije Universiteit Brussel (VUB), 1090 Brussels, Belgium \\ 3 Institute of Physics and Power Engineering (IPPE), Obninsk 249020, Russian Federation
}

\begin{abstract}
Excitation functions of the reactions ${ }^{\text {nat }} \operatorname{Er}(\alpha, \mathrm{x})^{167,169} \mathrm{Yb},{ }^{\text {nat }} \operatorname{Er}(\alpha, \mathrm{x}){ }^{172,173} \mathrm{Tm},{ }^{\text {nat }} \mathrm{Yb}(\alpha, \mathrm{x})^{170,173,175,177 \mathrm{~m} 2} \mathrm{Hf}$, ${ }^{\text {nat }} \mathrm{Yb}(\alpha, \mathrm{x}){ }^{171,172,177 \mathrm{~g}, 178 \mathrm{~m}} \mathrm{Lu}$ and ${ }^{\text {nat }} \mathrm{Yb}(\alpha, \mathrm{x}){ }^{169,177} \mathrm{Yb}$ were measured up to $38 \mathrm{MeV}$ for application purposes (medical isotope production and charged particle activation analysis). The experimental excitation functions are compared to the theoretical calculations based on the model code ALICE-IPPE. The literature is very poor regarding cross section measurements on erbium or ytterbium irradiated with alpha particles. Yields of different production routes (proton-, deuteron- and alpha-irradiation) of ${ }^{169} \mathrm{Yb}$ and ${ }^{177} \mathrm{Lu}$ are compared.
\end{abstract}

\section{Introduction}

Nowadays lots of radioactive isotopes are used in different fields, for example in industry, archaeology, biology and mainly in medicine. The practical importance of radionuclei ${ }^{169} \mathrm{Yb}$ and ${ }^{177} \mathrm{Lu}$ has been rising recently.

- ${ }^{169} \mathrm{Yb}(\mathrm{EC}=100 \%)$ is an almost pure Auger-electron and $\mathrm{X}$-ray emitter and has been gaining interest in brachytherapy, it has also found application in medical diagnostics, especially in cisternography and it is used in radiography of industrial materials.

- ${ }^{177} \mathrm{Lu}\left(\beta^{-}\right.$-decay) has excellent properties for applications in therapy and the emitted photons are also ideally suited for imaging and localisation with $\gamma$-cameras.

These medical radioisotopes are produced mainly via $(n, \gamma)$ reaction in reactors. However, in spite of the high reaction cross section, this production route yields a product of low specific activity and it is still not at the "no-carrier-added" level or chemical separation difficulties appear. Therefore, alternative production routes utilizing charged particle induced processes seemed to be interesting.

Excitation functions of the reactions ${ }^{\text {nat }} \operatorname{Er}(\alpha, \mathrm{x}){ }^{167,169} \mathrm{Yb}$, ${ }^{\text {nat }} \operatorname{Er}(\alpha, \mathrm{x}){ }^{172,173} \mathrm{Tm},{ }^{\text {nat }} \mathrm{Yb}(\alpha, \mathrm{x}){ }^{170,173,175,177 \mathrm{~m} 2} \mathrm{Hf},{ }^{\text {nat }} \mathrm{Yb}(\alpha, \mathrm{x})$ ${ }_{171,172,177 \mathrm{~g}, 178 \mathrm{~m}} \mathrm{Lu}$ and ${ }^{\mathrm{nat}} \mathrm{Yb}(\alpha, \mathrm{x})^{169,177} \mathrm{Yb}$ were measured up to $38 \mathrm{MeV}$ but here only results for ${ }^{169} \mathrm{Yb}$ and ${ }^{177} \mathrm{Lu}$ are presented. Earlier we investigated other charged particle induced production routes like ${ }^{169} \mathrm{Tm}(\mathrm{p}, \mathrm{n}){ }^{169} \mathrm{Yb}[1],{ }^{169} \mathrm{Tm}(\mathrm{d}, 2 \mathrm{n}){ }^{169} \mathrm{Yb}$ [2], ${ }^{176} \mathrm{Yb}(\mathrm{d}, \mathrm{p}){ }^{177} \mathrm{Yb} \rightarrow{ }^{177} \mathrm{Lu}$ and ${ }^{176} \mathrm{Yb}(\mathrm{d}, \mathrm{n}){ }^{177} \mathrm{Lu}$ [3] to determine their productivity. In this work yields for alphaproduction routes were calculated and yields of different production routes (proton-, deuteron- and alpha-irradiation) for ${ }^{169} \mathrm{Yb}$ and ${ }^{177} \mathrm{Lu}$ are compared.

\section{Experimental procedure}

Four alpha-irradiations were carried out at the external beam of CGR-560 cyclotron at Vrije Universiteit Brussel (VUB)

${ }^{a}$ Present author, e-mail: kiralyb@atomki.hu in Belgium, in 2006. Two stacks of natural erbium and two stacks of natural ytterbium target foils were irradiated, each separated by Ti monitor foils. At the beginning of one of the two stacks an Al degrader foil was placed to ensure a small energy shift. The thicknesses of the high purity foils are: Er: $32 \mu \mathrm{m}, \mathrm{Yb}: 30 \mu \mathrm{m}, \mathrm{Al}: 25.5 \mu \mathrm{m}, \mathrm{Ti}: 12 \mu \mathrm{m}$. The parameters of the irradiations are tabulated in table 1 .

Table 1. The parameters of the alpha-irradiations.

\begin{tabular}{ccccc}
\hline & \multicolumn{2}{c}{ Erbium } & \multicolumn{2}{c}{ Ytterbium } \\
& Stack1 & Stack2 & Stack1 & Stack2 \\
\hline Incident energy (MeV) & 37.5 & 37.9 & 38.5 & 39.0 \\
Beam current (nA) & 119.5 & 108.8 & 136.9 & 127.6 \\
Irradiation time (h) & 2.00 & 2.58 & 1.55 & 1.58 \\
\hline
\end{tabular}

Table 2. Decay data of radioisotopes studied [4].

\begin{tabular}{cccc}
\hline Nuclide & Half life & $\mathrm{E}_{\gamma}(\mathrm{keV})$ & $\mathrm{I}_{\gamma}(\%)$ \\
\hline${ }^{169 \mathrm{~g}} \mathrm{Yb}$ & $32.026 \mathrm{~d}$ & 130.5 & 11.31 \\
& & 177.2 & 22.16 \\
& & 307.7 & 10.05 \\
${ }^{177 g} \mathrm{Lu}$ & $6.734 \mathrm{~d}$ & 208.4 & 11 \\
\hline
\end{tabular}

No chemical separation was done. The $\gamma$-spectra of the target foils were registered by a PC-coupled HPGe detector system just after the end of bombardment and again a month later to get information for the radionuclei of short and long halflife as well and to follow cumulative processes. The sampledetector distance was chosen carefully to avoid pile-up effects and coincidence losses. The detector efficiency was determined placing standard sources at different distances from the detector.

\section{Data processing}

Decay data were taken from ref. [4] and are tabulated in table 2 . 
The mean bombarding energy of the beam in the subsequent foils was calculated following the polynomial approximation for stopping power of Andersen and Ziegler [5].

To specify the nominal incident energy and current of the alpha-beam, the cross sections of the ${ }^{\text {nat }} \mathrm{Ti}(\alpha, \mathrm{x})^{51} \mathrm{Cr}$ monitor reaction was re-measured over the whole energy range and adjusted to the curve recommended by the IAEA in ref. [6]. The excitation functions of monitor reactions for the ytterbium stacks are shown in figure 1.

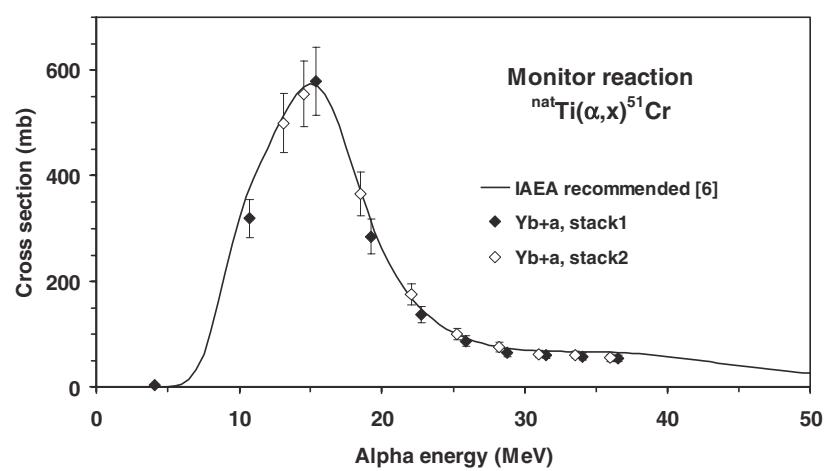

Fig. 1. Excitation function of the ${ }^{\mathrm{nat}} \mathrm{Ti}(\alpha, \mathrm{x})^{51} \mathrm{Cr}$ monitor reaction.

The energy uncertainty of the beam at the first foil is $\pm 0.3 \mathrm{MeV}$ which increases along the stack due to the energy straggling and the uncertainty of target thicknesses and reaches $\pm 1.5 \mathrm{MeV}$ at the last foil.

The uncertainties of the cross sections were determined in the standard way according to the guide of ref. [7]. The linearly contributing independent processes were taken into account extracting square root of quadratically summed relative uncertainties, namely: number of the bombarding particles $(7 \%)$, number of the target nuclei $(3 \%)$, decay data $(3 \%)$, detector efficiency $(7 \%)$ and peak area (1-10\%).

\section{Theoretical calculations}

The measured experimental data were compared to and analyzed with theoretical calculations based on the model code ALICE-IPPE. The ALICE code family was developed by Blann [8] and is based on the hybrid, the geometry dependent hybrid (GDH) or the hybrid Monte Carlo simulation (HMS) pre-equilibrium models and the Weisskopf-Ewing evaporation formalism. The ALICE-IPPE code [9] is a version of the ALICE-91 code [10] modified by the Obninsk group to include the generalized superfluid level density model and pre-equilibrium cluster emission. Corrections were made, among others, for $\gamma$-emission and optical model parameters. Angular distribution and refraction were not taken into account. The lack of angular momentum and parity treatments in the Weisskopf-Ewing formalism used makes independent treatment of isomeric states impossible, only total production cross sections can be calculated. The theoretical curves were determined using one recommended input data-set without any optimization or adjustment of parameters to the individual reactions or stable target isotopes.
The results of the reaction products of interest in this study were weighted and summed according to the abundance of the target isotopes and to the cumulative processes.

\section{Results and discussion}

The number of radionuclei cross sections which could be determined was restricted by the fact that both the erbium and ytterbium foils had already been irradiated with deuteron beams before. Four and ten excitation functions were measured after alpha-irradiations of stacks containing natural erbium and natural ytterbium target foils, respectively. Because of the limited space here we present only those which are of practical importance in nuclear medicine: ${ }^{169} \mathrm{Yb}$ and ${ }^{177} \mathrm{Lu}$. The rest of our results will be published separately soon.

\subsection{Cross sections}

${ }^{\text {nat }} \operatorname{Er}(\alpha, \mathrm{x})^{169} \mathrm{Yb}$ process. The cross sections were measured after complete IT decay of the short half-life $\left(\mathrm{T}_{1 / 2}=46 \mathrm{~s}\right)$ metastable state. Results are shown in figure 2. Two authors reported data measured on ${ }^{\text {nat }} \mathrm{Er}$ target. Data of Archenti et al. [11] are very scattered and therefore less reliable. The points of Sonzogni et al. [12] follow a curve that is similar to ours but are a factor of 3 lower. The ALICE-IPPE calculation describes well the shape of our experimental data but differs in absolute value.

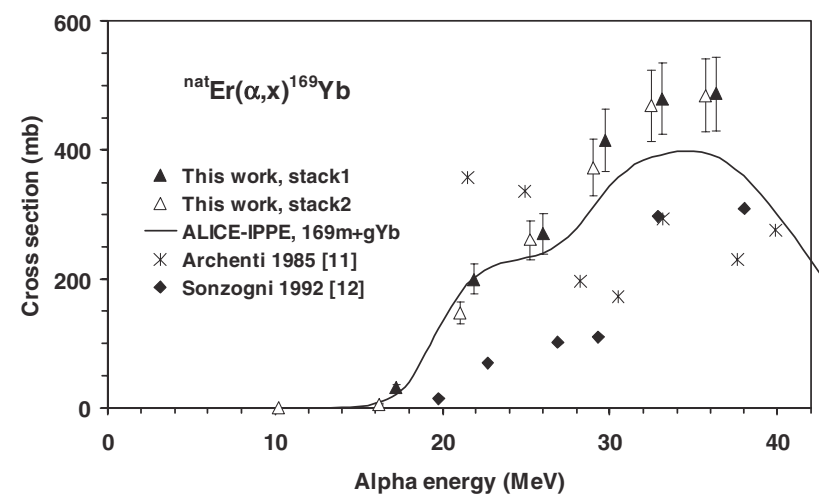

Fig. 2. Excitation function for ${ }^{\mathrm{nat}} \operatorname{Er}(\alpha, \mathrm{x})^{169} \mathrm{Yb}$ production.

${ }^{\text {nat }} \mathrm{Yb}(\alpha, \mathrm{x})^{169} \mathrm{Yb}$ cumulative process. This cumulative cross section includes contributions from total decay of ${ }^{169} \mathrm{Hf}\left(\mathrm{T}_{1 / 2}\right.$ $=3.24 \mathrm{~m}),{ }^{169 \mathrm{~m}} \mathrm{Lu}\left(\mathrm{T}_{1 / 2}=160 \mathrm{~s}\right),{ }^{169 \mathrm{~g}} \mathrm{Lu}\left(\mathrm{T}_{1 / 2}=34.06 \mathrm{~h}\right)$ and ${ }^{169 \mathrm{~m}} \mathrm{Yb}\left(\mathrm{T}_{1 / 2}=46 \mathrm{~s}\right)$ in addition to directly formed ${ }^{169 \mathrm{~g}} \mathrm{Yb}$. Only a few points could be determined because the maximum energy of our alpha-particles was only slightly above the effective threshold as figure 3 shows. The cross section axis is in logarithmic scale because the values vary over orders of magnitudes. Results of Romo et al. [13] are higher than ours in the overlapping energy range. However, we have to notice that as Romo used only one stack and the incident energy of the beam was $84 \mathrm{MeV}$, the uncertainty of the alpha energy at the end of the stack was large. The weak point of our results is the large uncertainty of the cross sections due 
to the poor statistics. Although the ALICE-IPPE calculation was not carried out to get results for ${ }^{169} \mathrm{Yb}$, the other reactions taking part in the cumulative process were added. The curve runs below the measured data.

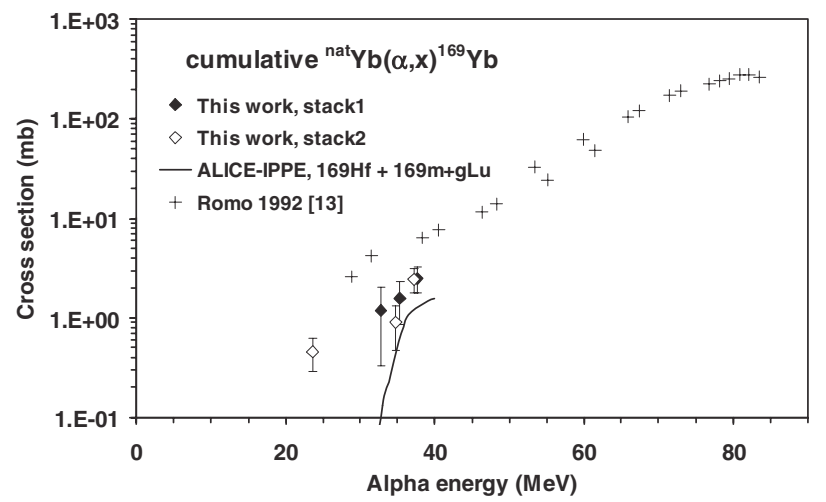

Fig. 3. Excitation function for cumulative ${ }^{\text {nat }} \mathrm{Yb}(\alpha, \mathrm{x})^{169} \mathrm{Yb}$ production.

${ }^{\text {nat }} \mathrm{Yb}(\alpha, \mathrm{x})^{177 \mathrm{~g}} \mathrm{Lu}$ cumulative process. This cumulative cross section may include contributions from ${ }^{177} \mathrm{Tm}\left(\mathrm{T}_{1 / 2}=85 \mathrm{~s}\right)$, ${ }^{177 m} \mathrm{Yb}\left(\mathrm{T}_{1 / 2}=6.41 \mathrm{~s}\right),{ }^{177 g} \mathrm{Yb}\left(\mathrm{T}_{1 / 2}=1.911 \mathrm{~h}\right)$ in addition to direct production of ${ }^{177 \mathrm{~g}} \mathrm{Lu}$ but not from ${ }^{177 \mathrm{~m}} \mathrm{Lu}\left(\mathrm{T}_{1 / 2}=\right.$ $160.4 \mathrm{~d}$ ) because its absence in the spectra was proved in the investigated energy range. No earlier data were found in the literature. We performed an ALICE-IPPE calculation only for ${ }^{177 m+g} \mathrm{Lu}$ so the curve goes below our measured points in figure 4.

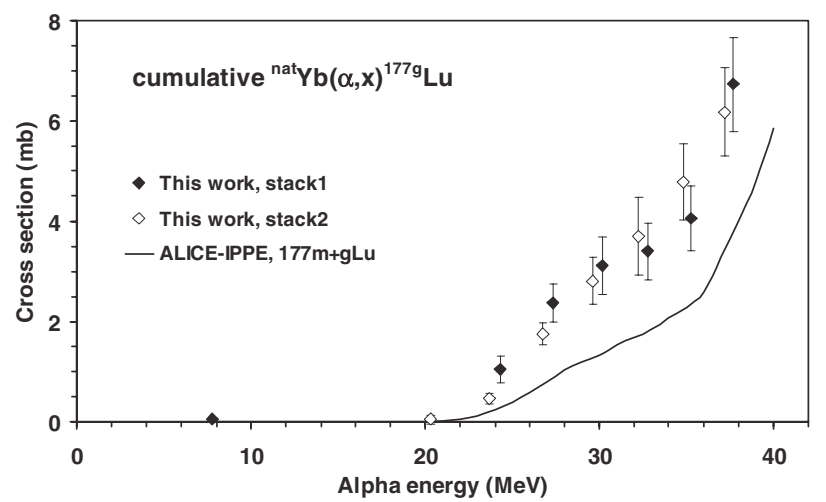

Fig. 4. Excitation function for cumulative ${ }^{\text {nat }} \mathrm{Yb}(\alpha, \mathrm{x})^{177 \mathrm{~g}} \mathrm{Lu}$ production.

\subsection{Integral yields}

The experimental cross sections were fitted and integral yields were calculated for ${ }^{169} \mathrm{Yb}$ and ${ }^{177} \mathrm{Lu}$ radioisotopes produced by alpha-irradiation on natural erbium and ytterbium targets. Results are shown in figures 5-6 compared to the integral yields calculated earlier on the basis of proton- and deuteronirradiations (see refs. [1-3]).

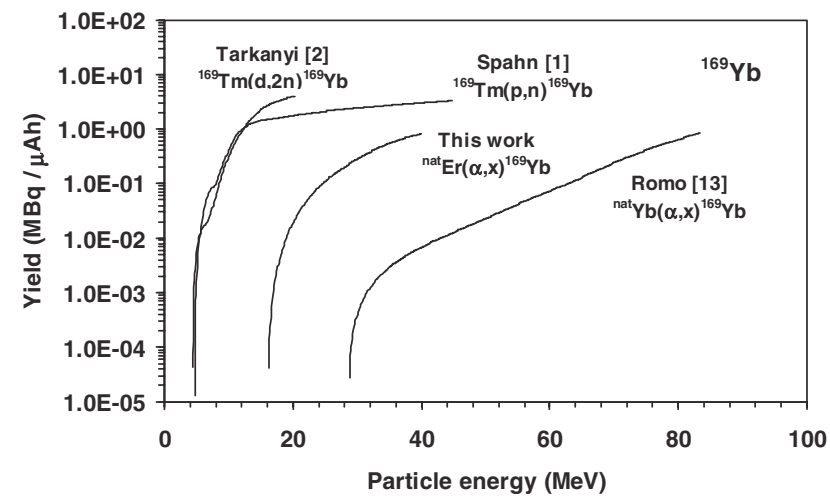

Fig. 5. Integral yields of ${ }^{169} \mathrm{Yb}$.

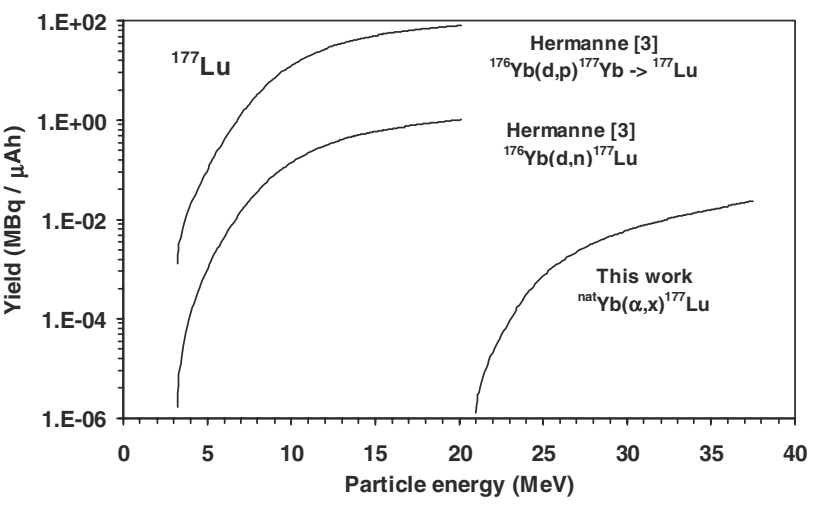

Fig. 6. Integral yields of ${ }^{177} \mathrm{Lu}$.

\section{Conclusion}

In the production of medical radioisotopes charged particle induced nuclear reactions can compete with neutron capture in reactors only if the aim is to reach higher specific activity and better quality product at "no-carrier-added" level. Comparing the proton-, deuteron- and alpha-irradiations on the targets studied we can conclude that the production of ${ }^{169} \mathrm{Yb}$ and ${ }^{177} \mathrm{Lu}$ by using an alpha-beam on natural erbium and natural ytterbium targets is not a defendable alternative.

\section{References}

1. I. Spahn et al., Appl. Radiat. Isot. 63, 235 (2005).

2. F. Tárkányi et al., Appl. Radiat. Isot. 65, 663 (2007).

3. A. Hermanne et al., Nucl. Instrum. Meth. B 247, 223 (2006).

4. L.P. Ekström, R.B. Firestone, WWW Table of Radioactive Isotopes, Database Version 2/28/99 from URL, http://ie.lbl.gov/toi/index.htm.

5. H.H. Andersen, J.F. Ziegler, Hydrogen stopping powers and ranges in all elements, Vol. 3 of The Stopping and Ranges of Ions in Matter (Pergamon Press, 1977), ISBN 0-08-021605-6.

6. F. Tárkányi et al., Beam monitor reactions (Ch. 4), in Charged Particle Cross-Section Database for Medical Radioisotope Production: Diagnostic Radioisotopes and Monitor Reactions, IAEA-TECDOC-1211, Vienna, 2001, p. 49. Available from: http://www-nds.indcentre.org.in/reports-new/tecdocs/iaeatecdoc-1211.pdf.

7. Guide to the Expression of Uncertainty in Measurement (International Organization for Standardization, Geneva, 1995), ISBN 92-67-10188-9. 
8. M. Blann, Recent Progress and Current Status of Preequilibrium Reaction Theories and Computer Code ALICE, LLNL Report, UCRL-JC-109052, 1991.

9. A.I. Dityuk et al., New Advanced Version of Computer Code ALICE-IPPE, Report INDC(CCP)-410 (International Atomic Energy Agency, Vienna, 1998).
10. M. Blann, ALICE-91, Report PSR-0146, available from: http://www.nea.fr/abs/html/psr-0146.html.

11. A. Archenti et al., Radiochim. Acta 38, 65 (1985).

12. A.A. Sonzogni et al., J. Radioanal. Nucl. Chem., Letters 165, 295 (1992).

13. A.S.M.A. Romo et al., Radiochim. Acta 57, 57 (1992). 